\title{
Near Optimal Power Allocation Algorithm for OFDM-Based Cognitive Using Adaptive Relaying Strategy
}

\author{
Hamza Soury ${ }^{\star}$, Faouzi Bader**, Musbah Shaat** and Mohamed-Slim Alouini ${ }^{\star}$ \\ $\star$ Electrical Engineering Program \\ King Abdullah University of Science and Technology (KAUST) \\ Thuwal, Makkah Province, Kingdom of Saudi Arabia \\ ** Centre Tecnologic de Telecommunication de Catalunya (CTTC) \\ Parc Mediterrani de la Tecnologia, Av. Carl Friedrich Gauss 7, 08860, Castelldefels-Barcelona, Spain \\ Email: \{soury.hamza, slim.alouini\}@kaust.edu.sa; \{faouzi.bader,musbah.shaat\}@cttc.es
}

\begin{abstract}
Relayed transmission increases the coverage and achievable capacity of communication systems. Adaptive relaying scheme is a relaying technique by which the benefits of the amplifying or decode and forward techniques can be achieved by switching the forwarding technique according to the quality of the signal. A cognitive Orthogonal Frequency-Division Multiplexing (OFDM) based adaptive relaying protocol is considered in this paper. The objective is to maximize the capacity of the cognitive radio system while ensuring that the interference introduced to the primary user is below the tolerated limit. A Near optimal power allocation in the source and the relay is presented for two pairing techniques such that the matching and random pairing. The simulation results confirm the efficiency of the proposed adaptive relaying protocol, and the consequence of choice of pairing technique.
\end{abstract}

\section{INTRODUCTION}

Recent measurement shows that the frequency spectrum is under-utilized by the licensed users, also called primary users (PUs). The spectrum utilization can be improved by allowing a group of secondary users (SUs) to use the vacant channels left by the PUs. Multicarrier communication has been suggested as a candidate for Cognitive Radio (CR) systems [1]. Such systems have to distribute their limited resources among the SUs in order to maximize the capacity without causing harmful interference to the PUs (see e.g. [2], [3]).

To increase coverage and achievable capacity of the communication system, relays ( $R$ ) are used to transfer the information from the cognitive source (CS) to the destination (D) when the direct link is not available. The resource allocation problem for the non-cognitive Orthogonal Frequency-Division Multiplexing (OFDM) based relay system has been widely studied [4], [5]. In [6], near optimal power allocation was derived for an OFDM based cognitive system with decode and forward relaying strategy.

In cooperative communication systems, Amplify-AndForward (AAF) and Decode-And-Forward (DAF) relaying strategies can be used. The AAF amplifies the received signal from the source $(S)$ then forward it to D. However, the relay working with the DAF strategy decodes "perfectly" the received signal from $S$ and then encodes it again and forwards it to D. The disadvantages of these two techniques of relaying come with the fact that the AAF relay amplifies the noise, which degrades the signal quality. On the other hand, the DAF relay causes an error propagation in case of incorrect decoding of the information symbols.

The adaptive relaying or Adaptive Relaying Protocol (ARP), as named in [4], is one of the proposed solutions to minimize the disadvantages of these two relaying techniques. In [5], the relay can execute $\mathrm{AAF}$ and $\mathrm{DAF}$, and there is a technique based on the Signal-to-Noise-Ratio (SNR) which trigger the switching between the AAF and DAF strategies. It assumes that at high SNR, the relay can decode perfectly so it works with DAF, and for low SNR, when it is harder to decode correctly it works with AAF.

In this paper, we integrate this technique of adaptive relaying in a CR based environment and we look at the power allocation at the relaying and the distribution of the subcarriers to the relay. The problem now is how to allocate optimally the power at the transmitters ( $\mathrm{S}$ and $\mathrm{R}$ ) to reach high capacity using ARP and without causing harmful interference to the primary user from the cognitive transmitters. We propose as a solutions of this problem an algorithm based on the subgradient method [7]. For simplicity, we selected, first, the subcarrier and assumed that the relay uses the same sub-carrier for receiving (from $\mathrm{S}$ ) and for transmission (to D) in second time slot, besides we used random selection of carrier from S-R to R-D and compared the performances of both schemes.

The rest of the paper is organized as follows. In Section II, we present the system model while the problem is formulated in Section III, and our proposed near optimal scheme is presented in section IV. Simulations and numerical results are discussed in Section V. Finally, the paper is concluded in Section VI.

\section{SYSTEM MODEL}

In this paper, an OFDM-based relay CR system is considered. The CR relay system coexists with the primary system 


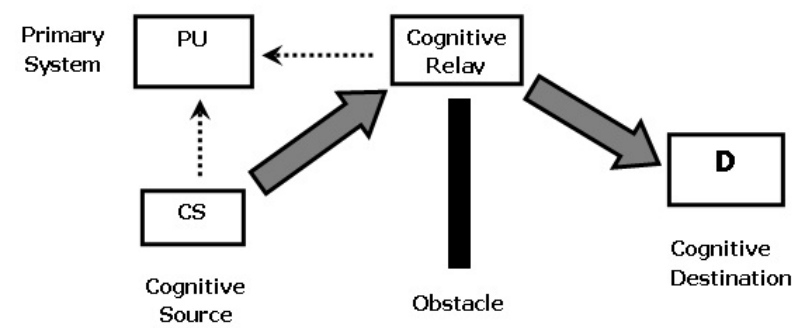

Fig. 1. Illustration of a cooperative relay cognitive radio network.

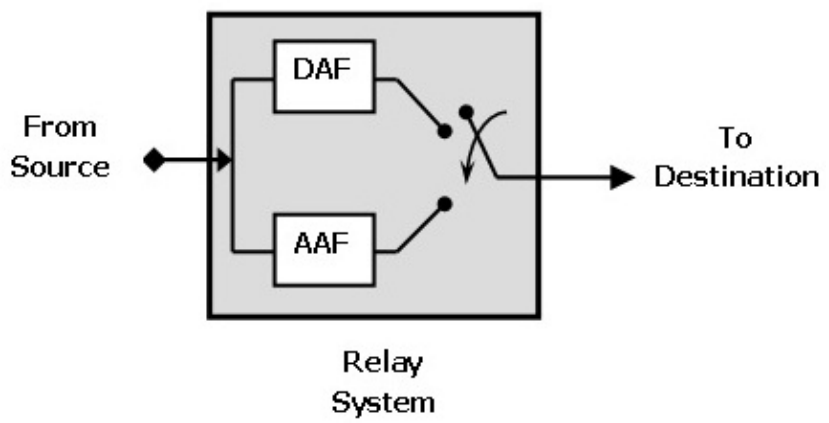

Fig. 2. Block diagram for the structure of an adaptive relay.

in the same geographical location. We suppose that there is no direct link between the cognitive source CS and the destination $\mathrm{D}$, so that $\mathrm{S}$ tries to communicate with $\mathrm{D}$ through the relay (see Fig.1). The frequency spectrum of the CR system is divided into $N$ subcarriers each having a $\Delta f$ bandwidth. We assume that the CR system can transmit through the unused PU band without exceeding the maximum interference power $I_{t h}$, that can be tolerated by PU. The relay receives and transmits in two different time slots. In the first time slot, $\mathrm{S}$ transmits to the Relay (R), while in the second time slot, $\mathrm{R}$ forwards the signal to $\mathrm{D}$ with ARP techniques. It has been assumed that we have one relay works with different channels. Note that the relay can forward the data using two techniques (DAF and AAF) by switching between them as presented in Fig.2.

The calculation of the mutual interference introduced by the $i^{t h}$ subcarrier to the PU and the interference power introduced by the PU signal into the band of the $i^{t h}$ subcarrier are given in [6].

\section{Problem Formulation}

Let us first define the variables of the problem. Let $\left(P_{S R}^{i} ; P_{R D}^{i}\right)$ be the power transmitted over the $i^{t h}$ subcarrier in the (S-R;R-D) link. The $i^{\text {th }}$ subcarrier channel gain over the (S-R;R-D) link is given by $\left(H_{S R}^{i} ; H_{R D}^{i}\right)$. Finally the noise variance is assigned to $\sigma_{i}^{2}=\sigma_{A W G N}^{2}+J_{i}$ where $\sigma_{A W G N}^{2}$ is the variance of the additive white Gaussian noise (AWGN) and $J_{i}$ is the interference introduced by the PU signal into the $i^{t h}$ subcarrier [6, Eq.3], since this interference can be modeled as AWGN as described in [2]. To make the analysis more clear, the noise variance is assumed to be the same for all subcarriers and both time slots $\sigma^{2}$.
Let $x_{s, i}$ be the transmitted signal from $\mathrm{S}$ over the $i^{t h}$ channel. The received signal at the relay $\mathbf{R}$ over the $i^{\text {th }}$ subcarrier in the first time slot is given by

$$
y_{S R}^{i}=\sqrt{H_{S R}^{i} P_{S R}^{i}} x_{s, i}+n_{S R}^{i},
$$

where $n_{S R}^{i}$ is the noise between $\mathrm{S}$ and $\mathrm{R}$ with variance $\sigma_{S R, i}^{2}=\sigma^{2}$.

According to the Shannon capacity formula, the transmission rate of the $i^{t h}$ subcarrier $R_{1, i}$ can be evaluated as

$$
R_{1, i}=\frac{1}{2} \log _{2}\left(1+\frac{P_{S R}^{i} H_{S R}^{i}}{\sigma^{2}}\right) .
$$

As it has been mentioned in the introduction, we should limit the interference caused by the CS to the PU, which give us the following interference constraint [6]

$$
\sum_{i=1}^{N} P_{S R}^{i} \Omega_{S P}^{i} \leq I_{t h},
$$

where $\Omega_{S P}^{i}$ denotes the interference factor of the $i^{t h}$ subcarrier to the PU band.

In the second time slot, the relay decodes and re-encodes or amplifies the signal over the $i^{t h}$ channel, depending on the received SNR, then forwards it to the destination. This means that the transmit signal from the relay over the $i^{t h}$ channel is

$$
x_{R D}^{i}= \begin{cases}\sqrt{P_{R D}^{i}} \cdot x_{s} & \text { for } P_{S R}^{i} \gamma_{S R}^{i} \geq \gamma_{t h} \\ \frac{\sqrt{P_{R D}^{i}} \cdot y_{S R}}{\sqrt{H_{S R}^{i} P_{S R}^{i}+\sigma^{2}}} & \text { for } P_{S R}^{i} \gamma_{S R}^{i}<\gamma_{t h},\end{cases}
$$

where $P_{S R}^{i} \gamma_{S R}^{i}=\frac{P_{S R}^{i} H_{S R}^{i}}{\sigma^{2}}$ is the received SNR via the sourcerelay link, and $\gamma_{t h}$ is the threshold SNR to ensure successful decoding. We assume that we have successful decoding when $P_{S R}^{i} \gamma_{S R}^{i}$ is above $\gamma_{t h}$.

At the destination the received signal over the $i^{\text {th }}$ channel can be written as

$$
y_{R D}^{i}=\sqrt{H_{R D}^{i}} x_{R D}^{i}+n_{R D}^{i} .
$$

Let us define two variables: $\gamma_{S R}^{i}=\frac{H_{S R}^{i}}{\sigma^{2}}$ and $\gamma_{R D}^{i}=\frac{H_{R D}^{i}}{\sigma^{2}}$. Using (4) and (5), we derive the expression of the total SNR delivered via the $i^{\text {th }}$ channel as

$$
\gamma_{A R P}^{i}= \begin{cases}P_{R D}^{i} \gamma_{R D}^{i} & \text { for } P_{S R}^{i} \gamma_{S R}^{i} \geq \gamma_{t h} \\ \gamma_{A F}^{i} & \text { for } P_{S R}^{i} \gamma_{S R}^{i}<\gamma_{t h},\end{cases}
$$

where $\gamma_{A F}^{i}$ is the SNR for the set of channels that work on amplify-and-forward, and it is given by

$$
\gamma_{A F}^{i}=\frac{P_{S R}^{i} \gamma_{S R}^{i} P_{R D}^{i} \gamma_{R D}^{i}}{P_{R D}^{i} \gamma_{R D}^{i}+P_{S R}^{i} \gamma_{S R}^{i}+1} .
$$

Back to the capacity, using Shannon formula, we calculate the rate of the channel in the second time slot for the two cases as

$$
\left\{\begin{array}{c}
R_{2, D A F, i}=\frac{1}{2} \log _{2}\left(1+\frac{P_{R D}^{i} H_{R D}^{i}}{\sigma^{2}}\right) \text { for the DAF case } \\
R_{2, A A F, i}=\frac{1}{2} \log _{2}\left(1+\frac{P_{R D}^{i} \gamma_{R D}^{i} P_{S R}^{i} \gamma_{S R}^{i}}{P_{R D}^{i} \gamma_{R D}^{i}+P_{S R}^{i} \gamma_{S R}^{i}+1}\right) \\
\text { for the AAF case. }
\end{array}\right.
$$


Note that $R_{2, A A F, i}$ is not jointly concave in $P_{R D}^{i}$ and $P_{S R}^{i}$. To make the analysis simpler, we adopt the following approximation

$$
R_{2, A A F, i} \approx \frac{1}{2} \log _{2}\left(1+\frac{P_{R D}^{i} \gamma_{R D}^{i} P_{S R}^{i} \gamma_{S R}^{i}}{P_{R D}^{i} \gamma_{R D}^{i}+P_{S R}^{i} \gamma_{S R}^{i}}\right) .
$$

This approximation is used in [8] and is based on the assumption that the system has a high SNR for the amplified signal between the relay and the destination. It is proved in [9] that this approximation is also accurate even at moderate-low SNR regime.

Thus the transmission rate is given by

$R_{i}=\alpha_{i} \min \left\{R_{1, i}, R_{2, D A F, i}\right\}+\left(1-\alpha_{i}\right) \min \left\{R_{1, i}, R_{2, A A F, i}\right\}$

where $\alpha_{i}$ take the values "0" or " 1 " and indicate if the relay use DAF technique $\left(\alpha_{i}=0\right)$ or $\operatorname{AAF}$ technique $\left(\alpha_{i}=1\right)$.

We denote by $\mathcal{A}$ the set of index of channels that work on $\mathrm{AAF}$, and $\mathcal{D}$ as the set of index of channels that work on DAF

$$
\mathcal{A}=\left\{i, \alpha_{i}=1\right\} ; \mathcal{D}=\left\{i, \alpha_{i}=0\right\} .
$$

As in the DAF case, we should compute the interference caused by the relay to the PU for the AAF case. An expression given in [10, Eq.17-18] is adopted for our system, and we get the following interference constraint in the $2^{\text {nd }}$ time slot $(R \longrightarrow D)$

$$
\sum_{i \in \mathcal{A}} P_{R D}^{i} \Omega_{R P}^{i}+\sum_{i \in \mathcal{D}} P_{R D}^{i} \Omega_{R P}^{i} \leq I_{t h} .
$$

Our objective is to maximize the total capacity of the CR system while the interference introduced to the primary user is below the tolerated threshold. Therefore the optimization problem can be formulated as follows

$$
\max _{P_{S R}^{i}, P_{R D}^{i}} \sum_{i=1}^{N} R_{i}
$$

Subject to - (Interference at first time slot)

$$
\begin{gathered}
\sum_{i=1}^{N} P_{S R}^{i} \Omega_{S P}^{i} \leq I_{t h} ; \quad P_{S R}^{i} \geq 0 \\
- \text { (Interference at second time slot) } \\
\sum_{i \in \mathcal{A}} P_{R D}^{i} \Omega_{R P}^{i}+\sum_{i \in \mathcal{D}} P_{R D}^{i} \Omega_{R P}^{i} \leq I_{t h} ; \quad P_{R D}^{i} \geq 0 .
\end{gathered}
$$

In this problem, the power constraints at each transmitter ( $\mathrm{S}$ and R) are missing. However, when we take a look at the interference constraints, we note that the power constraint is included. Moreover, we use the identity $\Omega_{S P}^{i} \geq \min _{j} \Omega_{S P}^{j}$ to ensure the following inequality $\sum_{i=1}^{N} P_{S R}^{i} \leq \frac{I_{t h}}{\min _{j} \Omega_{S P}^{j}}$. Thus the interference constraint implies, indirectly, the power constraint in the two time slots.

To solve this problem, we assume that all fading gains are perfectly known. The channel gains between the CR system parts (,,$R$ and $D)$ can be obtained by channel estimation techniques, the channel gains between the CR system and the
PU can be obtained by estimating the received signal power from the primary terminal when it transmits [11].

From (10), the maximum capacity over the $i^{\text {th }}$ subcarrier can be achieved when

$$
R_{1, i}= \begin{cases}R_{2, D A F, i} & \text { for } i \in \mathcal{D} \\ R_{2, A A F, i} & \text { for } i \in \mathcal{A}\end{cases}
$$

Using (14),(2) and (8) we derive the following relation between the transmission powers

$$
P_{R D}^{i}=\frac{P_{S R}^{i} H_{S R}^{i}}{H_{R D}^{i}} \quad \text { for } i \in \mathcal{D} .
$$

In the other hand, if we look to the formula of the rate in the AAF case (8), we can see that the rate in the second time slot is always less than the rate in the first time slot and cannot reach it, in fact we have

$$
\frac{P_{R D}^{i} \gamma_{R D}^{i} P_{S R}^{i} \gamma_{S R}^{i}}{P_{R D}^{i} \gamma_{R D}^{i}+P_{S R}^{i} \gamma_{S R}^{i}}<\frac{P_{S R}^{i} H_{S R}^{i}}{\sigma^{2}} \quad \text { for } i \in \mathcal{A} .
$$

This means that the maximum rate of these channels equal to the rate in the second time slot. According to these derivations, we find the total expression of the rate in our model as

$$
\begin{aligned}
& R=\sum_{i \in \mathcal{A}} \frac{1}{2} \log _{2}\left(1+\frac{P_{R D}^{i} \gamma_{R D}^{i} P_{S R}^{i} \gamma_{S R}^{i}}{P_{R D}^{i} \gamma_{R D}^{i}+P_{S R}^{i} \gamma_{S R}^{i}}\right) \\
&+\sum_{i \in \mathcal{D}} \frac{1}{2} \log _{2}\left(1+P_{S R}^{i} \gamma_{S R}^{i}\right) .
\end{aligned}
$$

By assembling the previous equations, we can re-write the optimization problem given in (13) as

$$
\begin{gathered}
\max _{P_{S R}^{i}, P_{R D}^{i}} \frac{1}{2} \sum_{i \in \mathcal{A}} \log _{2}\left(1+\frac{P_{R D}^{i} \gamma_{R D}^{i} P_{S R}^{i} \gamma_{S R}^{i}}{P_{R D}^{i} \gamma_{R D}^{i}+P_{S R}^{i} \gamma_{S R}^{i}}\right) \\
+\frac{1}{2} \sum_{i \in \mathcal{D}} \log _{2}\left(1+P_{S R}^{i} \gamma_{S R}^{i}\right) \\
\text { s.t. } \quad \sum_{i=1}^{N} P_{S R}^{i} \Omega_{S P}^{* i} \leq I_{t h} ; \\
\sum_{i \in \mathcal{A}} P_{R D}^{i} \Omega_{R P}^{i}+\sum_{i \in \mathcal{D}} \frac{P_{S R}^{i} H_{S R}^{i}}{H_{R D}^{i}} \Omega_{R P}^{i} \leq I_{t h} ; \\
P_{S R}^{i} \geq 0 ; \\
P_{R D}^{i} \geq 0 .
\end{gathered}
$$

Under the previous assumption of perfect knowledge of the channel coefficient and the noise variance the problem is a convex optimization problem with the parameter $P_{R D}^{i}$ and $P_{S R}^{i}$. In the next section, we solve this problem using the Lagrangian method and the KKT conditions.

\section{Problem Solution}

For simplicity reasons, and for making the mathematical notation easy to follow, we denote, in this section, $P_{S R}^{i}$ by $P_{1}^{i}, P_{R D}^{i}$ by $P_{2}^{i}, \gamma_{S R}^{i}$ by $\gamma_{1}^{i}, \gamma_{R D}^{i}$ by $\gamma_{2}^{i}, \Omega_{S P}^{i}$ by $\Omega_{i}^{*}$ and $\Omega_{R P}^{i}$ by $\Omega_{i}$. 


\section{A. Dual Problem}

The Lagrangian function with Lagrangian multipliers $\lambda, \beta$ can be written as

$$
\begin{gathered}
\mathcal{L}=\frac{1}{2} \sum_{i=1}^{N}\left[\alpha_{i} \log _{2}\left(1+\frac{P_{1}^{i} \gamma_{1}^{i} P_{2}^{i} \gamma_{2}^{i}}{P_{1}^{i} \gamma_{1}^{i}+P_{2}^{i} \gamma_{2}^{i}}\right)\right. \\
\left.+\left(1-\alpha_{i}\right) \log _{2}\left(1+P_{1}^{i} \gamma_{1}^{i}\right)\right]+\beta\left(I_{t h}-\sum_{i=1}^{N} P_{1}^{i} \Omega_{i}^{*}\right) \\
+\lambda\left(I_{t h}-\sum_{i=1}^{N} \alpha_{i} P_{2}^{i} \Omega_{i}+\left(1-\alpha_{i}\right) \frac{P_{1}^{i} H_{S R}^{i}}{H_{R D}^{i}} \Omega_{i}\right) .
\end{gathered}
$$

Note that we substitute $\mathcal{A}$ and $\mathcal{D}$ by their definition and we include $\alpha_{i}$ in the Lagrangian to simplify the computation. We develop the Lagrangian to get the following expression

$$
\begin{gathered}
\mathcal{L}=\sum_{i=1}^{N} \alpha_{i}\left[\frac{1}{2} \log _{2}\left(1+\frac{P_{1}^{i} \gamma_{1}^{i} P_{2}^{i} \gamma_{2}^{i}}{P_{1}^{i} \gamma_{1}^{i}+P_{2}^{i} \gamma_{2}^{i}}\right)-\lambda P_{2}^{i} \Omega_{i}-\beta P_{1}^{i} \Omega_{i}^{*}\right] \\
+\left(1-\alpha_{i}\right)\left[\frac{1}{2} \log _{2}\left(1+P_{1}^{i} \gamma_{1}^{i}\right)-\lambda \frac{P_{1}^{i} H_{S R}^{i}}{H_{R D}^{i}} \Omega_{i}-\beta P_{1}^{i} \Omega_{i}^{*}\right] \\
+(\lambda+\beta) I_{t h} .
\end{gathered}
$$

Now we solve this problem by using the dual approach. Let us first define the dual problem as

$$
\min _{\beta \geq 0, \lambda \geq 0} g(\beta, \lambda)
$$

where

$$
g(\beta, \lambda) \triangleq \max _{P_{1}^{i}, P_{2}^{i}, \alpha_{i}} \mathcal{L} .
$$

From (20), and for a given set of $\alpha_{i}$, the problem can be divided into $N$ independent problems, and $\alpha_{i}$ has two values (0 or 1$)$ for all the subcarrier $i$.

For each subcarrier $i$, and given $\lambda$ and $\beta$, there are two cases:

- Case $\alpha_{i}=1$

The problem can be written as follows

$$
\begin{aligned}
g_{i}(\beta, \lambda)= & \max _{P_{1}^{i}, P_{2}^{i} \geq 0} \mathcal{L}_{1} \\
= & \max _{P_{1}^{i}, P_{2}^{i} \geq 0} \frac{1}{2} \log _{2}\left(1+\frac{P_{1}^{i} \gamma_{1}^{i} P_{2}^{i} \gamma_{2}^{i}}{P_{1}^{i} \gamma_{1}^{i}+P_{2}^{i} \gamma_{2}^{i}}\right) \\
& -\lambda P_{2}^{i} \Omega_{i}-\beta P_{1}^{i} \Omega_{i}^{*}
\end{aligned}
$$

which leads to

$$
\frac{\partial \mathcal{L}_{1}}{\partial P_{1}^{i}}=\frac{\left(P_{2}^{i}\right)^{2}\left(\gamma_{2}^{i}\right)^{2} \gamma_{1}^{i}}{\left(P_{2}^{i} \gamma_{2}^{i}+P_{1}^{i} \gamma_{1}^{i}\right)\left(P_{2}^{i} \gamma_{2}^{i}+P_{1}^{i} \gamma_{1}^{i}+P_{2}^{i} \gamma_{2}^{i} P_{1}^{i} \gamma_{1}^{i}\right)}-\beta \Omega_{i}^{*}
$$

$$
\frac{\partial \mathcal{L}_{1}}{\partial P_{2}^{i}}=\frac{\left(P_{1}^{i}\right)^{2}\left(\gamma_{1}^{i}\right)^{2} \gamma_{2}^{i}}{\left(P_{2}^{i} \gamma_{2}^{i}+P_{1}^{i} \gamma_{1}^{i}\right)\left(P_{2}^{i} \gamma_{2}^{i}+P_{1}^{i} \gamma_{1}^{i}+P_{2}^{i} \gamma_{2}^{i} P_{1}^{i} \gamma_{1}^{i}\right)}-\lambda \Omega_{i}
$$

Then we equalize (23) and (24) to zero. The solution of these equations leads to $P_{1}^{i}=c_{i} P_{2}^{i}$, where $c_{i}=\sqrt{\frac{\gamma_{2}^{i} \lambda \Omega_{i}}{\gamma_{1}^{2} \beta \Omega_{i}^{*}}}$. Thus the new value of $P_{2}^{i}$ is

$$
P_{2}^{i}=\left[\frac{\gamma_{2}^{i}}{\beta c_{i} \Omega_{i}^{*}\left(\gamma_{2}^{i}+c_{i} \gamma_{1}^{i}\right)}-\frac{1}{c_{i} \gamma_{1}^{i}}-\frac{1}{\gamma_{2}^{i}}\right]^{+}
$$

where $[x]^{+}=\max (0, x)$.

- Case $\alpha_{i}=0$

In this case (DAF) the problem has been solved in [6]. We have just to know $P_{1}^{i}$ and we have the relation $P_{2}^{i}=\frac{P_{1}^{i} H_{S R}^{i}}{H_{R D}^{i}}$. The solution is given, in this case, by the following expression

$$
P_{1}^{i}=\left[\frac{1}{\beta \Omega_{i}^{*}+\lambda \frac{H_{S R}^{i}}{H_{R D}^{i}} \Omega_{i}}-\frac{1}{\gamma_{1}^{i}}\right]^{+} .
$$

To solve this problem we use the subgradient algorithm [7].

\section{B. Sub-gradient Method to Solve the Dual Problem}

With the obtained optimal values of primal variables $\left(P_{1}^{i}\right.$, $P_{2}^{i}$ ), the dual problem can be solved using the sub-gradient method [7], [12], [13]. In fact our algorithm is based on the calculation of the Lagrangian multipliers, $\lambda$ and $\beta$, in each iteration. The decision about the type of relaying mode over each subcarrier is made using (6). The implementation procedures is described in Algorithm 1.

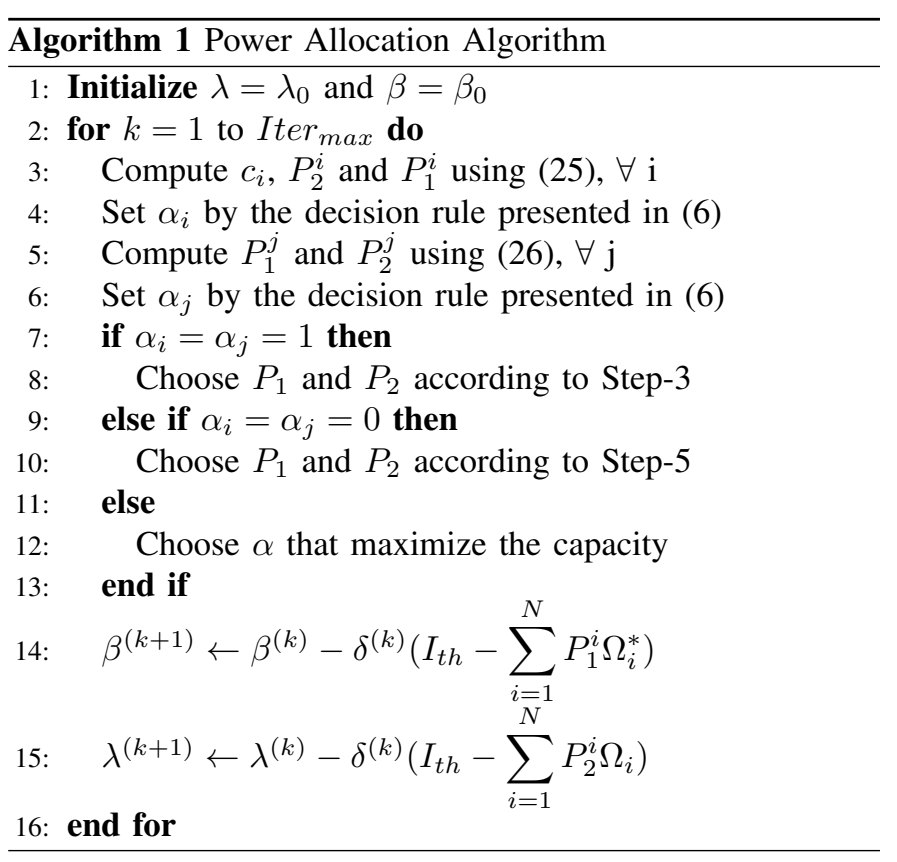

where $\delta^{(k)}$ is the step size of the $k^{t h}$ iteration.

This algorithm is well described in [7], [12], [13], where many types of step size can be used in the sub-gradient algorithm. In our model we try different step sizes and then use the best one in terms of best results and less complexity.

\section{Simulation And Results}

The simulations are performed under the scenario given in Section (II). An OFDM system of $N$ subcarriers $(N \in$ $\{16,32,64\})$ at the source and destination and one relay system is assumed. The values of $T_{s}, \Delta f$ and $I_{t h}$ are assumed to be $4 \mu$ seconds, $0.3125 \mathrm{MHz}$ and $-20 \mathrm{dBm}$ respectively. The channel gains are outcomes of independent Rayleigh distributed random variables with mean equal to 1 . 


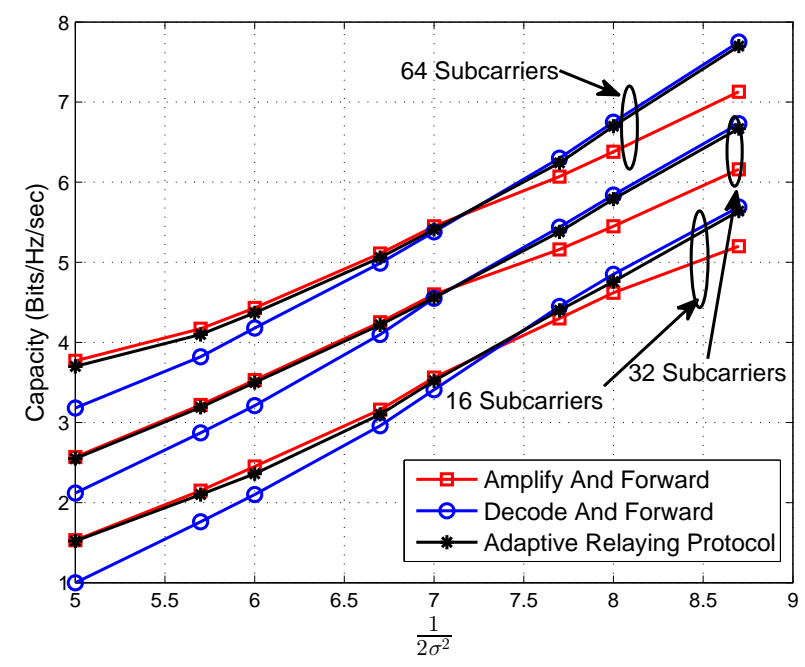

Fig. 3. Achieved capacity for different SNR, different number of sub-carriers, and $I_{t h}=10^{-5} W$

Fig. 3 plots the average capacity using the different schemes (AAF, DAF,ARP) vs. the SNR and using different values of total number of subcarriers with $N=\{16,32,64\}$. It is shown that for low values of SNR $\left(\frac{1}{2 \sigma^{2}}<7\right)$ and for each value of $N$, the DAF relay decoding procedure is not perfect. Therefore the AAF performs better than the DAF and provides higher capacity. However, at high SNR $(>7)$ values the behavior of the system become inverse to the previous situation. Here, decoding can be done "perfectly" and the propagation of errors due to the amplification in AAF process has more chances to occur. Thus, in this SNR region the performance achieved by the DAF process is higher than that achieved by the AAF.

It can be also shown that the ARP relaying protocol achieves for the different depicted values of SNR the best results. This can be explained by the fact that the ARP protocol is able to switch (in an adaptive way) from one relaying mode to another (AAF or DAF) using in each moment the relaying mode that achieves the best performance. In other words, the ARP tends to use the AAF relaying protocol for low values of SNR, and use the DAF for higher SNRs. Thus, ARP is able to take advantage of each relaying mode depending on the SNR range.

In terms of number of subcarriers, Fig.3 shows that the system capacity growth by increasing the total number of carriers of the system.

Fig.4 depicts the average capacity using all the relaying schemes (AAF, DAF, ARP) having two interference threshold values which are $10^{-5} \mathrm{~W}$ and $3 \times 10^{-5} \mathrm{~W}$. It can be shown that the crossing point between the DAF and the AAF curves occurs at different SNR values when the system has different interference constraints. Note that, for high values of interference threshold, the source and the relay will be able to transmit with more power than with small interference constraints $\left(I_{t h}\right.$

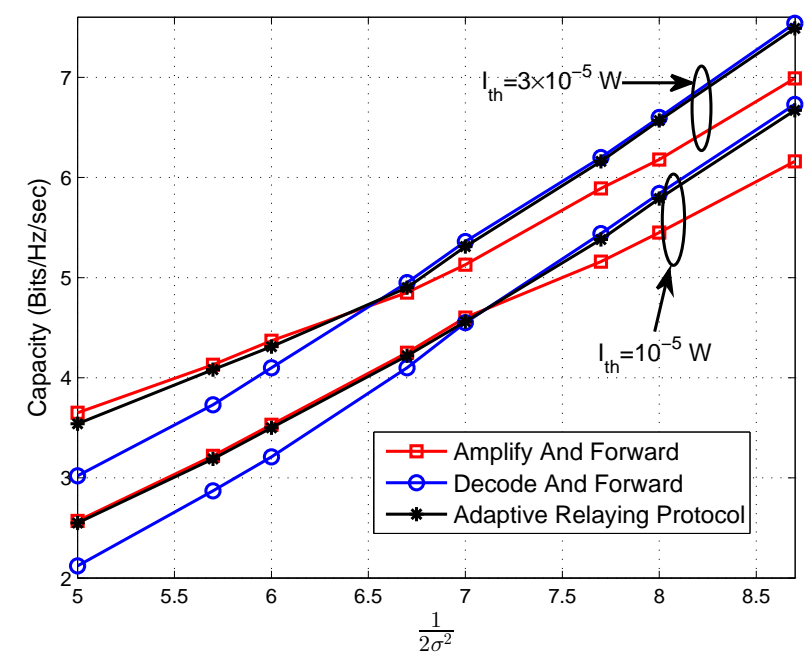

Fig. 4. Achieved capacity for different SNR, 32 subcarriers, and two values of the interference threshold: $I_{t h}=10^{-5} \mathrm{~W}$ and $I_{t h}=3.10^{-5} \mathrm{~W}$.

is small). This result implies that the decode procedure can be done correctly at the relay phase even for high noise variance.

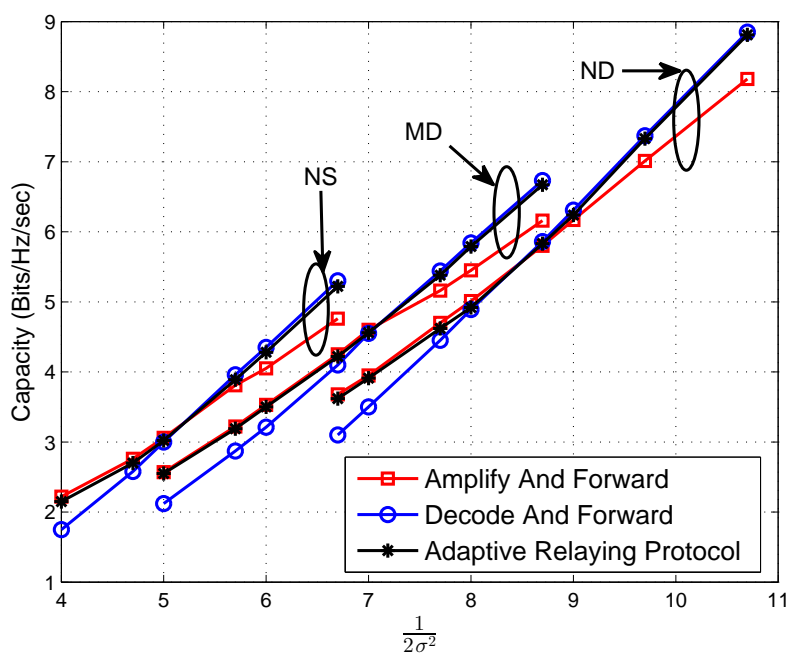

Fig. 5. Achieved capacity for different SNR, different position of the relays, $I_{t h}=10^{-5} \mathrm{~W}$ and 32 subcarriers: NS: Near Source, MD: Middle Distance, ND: Near Destination

Fig.5 shows the capacity of the system versus the SNR having the relay system at different distances from the source. A general observation is that the ARP achieves higher capacity when relay is near the destination, then the performances decrease as soon as we have the relay at middle distance between the $\mathrm{S}$ and $\mathrm{D}$, and near to the source respectively. It can be shown that the crossing point between the use of the AAF or the DAF appears at lower values of SNR if the relay system is located near the source. This can be explained by 
the fact that the relay receives at high SNR then the decoding can be done correctly so the relay switches to DAF. However, when the relay is near the destination the intersection point appears at high SNR. In this case, the received signal at the relay has low SNR, then the amplify and forward process of the signal perform better than the decode and forward for low and moderate values of SNR.

As a general observation from Figures 3, 4 and 5, it can be shown that the ARP scheme behavior reaches always the optimal scheme for different SNR values. However the major limitation of the proposed scheme is its complexity. Thus, new algorithm with much less complexity is required to make a step towards real implementation. Further work will be the development of suboptimal algorithm that achieves a near optimal performance with affordable complexity of implementation.

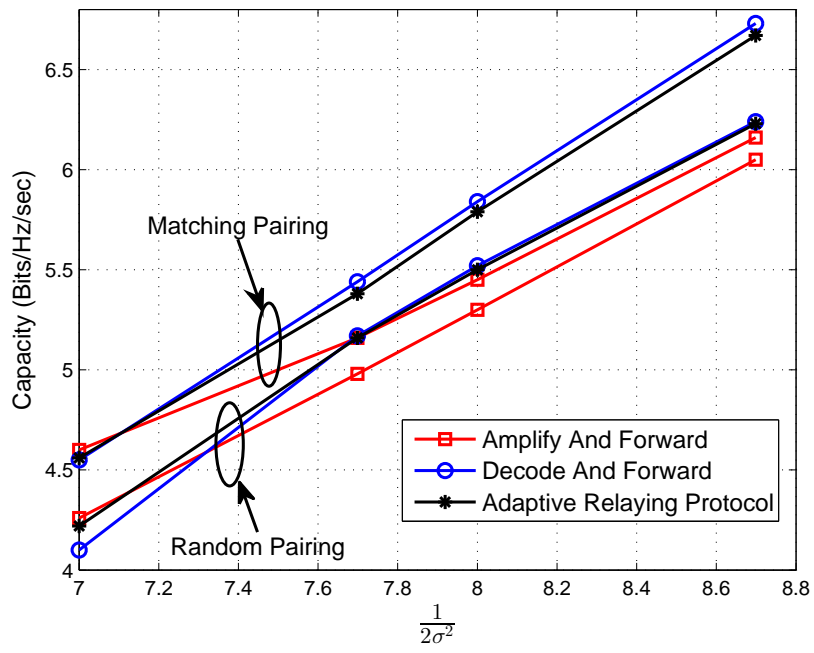

Fig. 6. Achieved capacity for different SNR, $I_{t h}=10^{-5} \mathrm{~W}, 32$ subcarriers and two types of subcarrier pairing: subcarrier matching and Random pairing

Fig.6 shows capacity performance comparison using the matching and random pairing techniques for different values of SNR. In matching pairing technique, the same carrier $k$ is used in both time slots ( in S-R and R-D links). However, with random pairing technique, assigned carrier in the second time slot will be chosen randomly. It can be shown in this figure, that higher capacity is achieved by matching carriers pairing than using the random assignment process of the carriers from S-R to R-D . It can be also observed that the ARP relaying technique achieves best performances in both cases; matching, and random pairing for different values of SNRs.

We can conclude that using the matching pairing technique with ARP relaying strategy, higher capacity performance could be achieved for a wide range of SNR values. However, the authors will focus on their further works on choosing the best pairing strategy to improve obtained results.

\section{CONCLUSiON}

In this paper, a near optimal power allocation algorithm in cognitive radio OFDM-based with adaptive relaying protocol is presented. The complexity of this algorithm is that we allocate jointly the power in source and relay by ensuring the interference constraint and choose the type of relay in an adaptive way. This algorithm distributes jointly the power in source and relay so that no excessive interference is introduced to the primary user. The proposed algorithm achieves a near optimal performance, which depends largely on the pairing technique. We are currently working on the extension of the proposed system by considering multiple relay nodes using adaptive carrier pairing strategy.

\section{ACKNOWLEDMENTS}

This work was partially supported by the European project ACROPOLIS-NoE (ICT-2009.1.1), the COST Action IC0902 and in part by a King Abdullah University of Science and Technology fellowship.

\section{REFERENCES}

[1] S. Haykin, "Cognitive radio: Brain-empowered wireless communications," IEEE Journal on Selected Areas in Communications, vol. 23 , no. 2, pp. $201-220$, Feb. 2005.

[2] G. Bansal, M. Hossain, and V. Bhargava, "Optimal and suboptimal power allocation schemes for OFDM-based cognitive radio systems," IEEE Transactions on Wireless Communications, vol. 7, no. 11, pp. $4710-4718$, November 2008.

[3] M. Shaat and F. Bader, "Computationally efficient power allocation algorithm in multicarrier-based cognitive radio networks: OFDM and FBMC systems," EURASIP Journal on Advances in Signal Processing, vol. 2010, 2010.

[4] Y. Li and B. Vucetic, "On the performance of a simple adaptive relaying protocol for wireless relay networks," in Proc. IEEE Vehicular Technology Conference, (VTC2008-Spring), Singapore, May 2008, pp. $2400-2405$

[5] A. Khan and V. Kühn, "Power optimization in adaptive relay networks," in Proc. IEEE Global Telecommun. Conf. (GLOBECOM 2010), Miami, Florida, USA, Dec. 2010.

[6] M. Shaat and F. Bader, "Optimal power allocation algorithm for OFDMbased decode-and-forward dual-hop cognitive systems," in Proc. IEEE 73rd Vehicular Technology Conference (VTC2011-Spring), Budapest, Hungary, May 2011.

[7] N. Z. Shor, K. C. Kiwiel, and A. Ruszcayǹski, Minimization Methods for Non-differentiable Functions. New York, NY, USA: Springer-Verlag New York, Inc., 1985.

[8] W. Dang, M. Tao, H. Mu, and J. Huang, "Subcarrier-pair based resource allocation for cooperative multi-relay OFDM systems," IEEE Transactions on Wireless Communications, vol. 9, no. 5, pp. 1640 -1649, May 2010.

[9] J. Tang and X. Zhang, "Cross-layer resource allocation over wireless relay networks for quality of service provisioning," IEEE Journal on Selected Areas in Communications, vol. 25, no. 4, pp. 645 -656, May 2007.

[10] W. Yue, B. Zheng, and Q. Meng, "Optimal power allocation for cognitive relay networks," in Proc. International Conference on Wireless Communications Signal Processing (WCSP 2009), Nanjing, China, Nov. 2009, pp. $1-5$

[11] R. Zhang, S. Cui, and Y.-C. Liang, "On ergodic sum capacity of fading cognitive multiple-access and broadcast channels," IEEE Transactions on Information Theory, vol. 55, no. 11, pp. 5161 -5178, Nov. 2009.

[12] M. Akgül, Topics in Relaxation and Ellipsoidal Methods, ser. RESEARCH NOTES IN MATHEMATICS SERIES. Pitman Advanced Pub. Program, 1984, vol. 97.

[13] D. P. Bertsekas, Nonlinear Programming, 2nd ed. Athena Scientifc, 1999. 